\title{
A Study on the Optoelectronics Parameters of Natural Dyes Extracted From Beetroot, Cabbage, Walnut and Henna For Potential Applications in Organic Electronics
}

\section{Peshawa 0. Amin}

Charmo University

Fahmi F. Muhammadsharif ( $\nabla$ fahmi982@gmail.com)

Koya University https://orcid.org/0000-0002-4563-9671

\section{Salah Raza Saeed \\ Charmo University}

Khaulah Sulaiman

Universiti Malaya

\section{Research Article}

Keywords: optoelectronic parameters, natural dyes, beetroot, cabbage, walnut, henna

Posted Date: July 29th, 2021

DOI: https://doi.org/10.21203/rs.3.rs-732502/v1

License: (c) (i) This work is licensed under a Creative Commons Attribution 4.0 International License.

Read Full License

Version of Record: A version of this preprint was published at Journal of Fluorescence on October 25th, 2021. See the published version at https://doi.org/10.1007/s10895-021-02837-7. 


\title{
A study on the optoelectronics parameters of natural dyes extracted from beetroot, cabbage, walnut and henna for potential applications in organic electronics
}

\author{
Peshawa O. Amin ${ }^{1}$, Fahmi F. Muhammadsharif ${ }^{2, *}$, Salah Raza Saeed ${ }^{1}$, and Khaulah Sulaiman ${ }^{3}$ \\ (1) Charmo Center for Research, Training and Consultancy, Charmo University, 46023 Chamchamal, Kurdistan Region, Iraq \\ (2) Department of Physics, Faculty of Science and Health, Koya University, Koya KOY45, Kurdistan Region - F.R., Iraq \\ (3) Low Dimensional Materials Research Centre (LDMRC), Department of Physics, Faculty of Science, Universiti Malaya, 50603 \\ Kuala Lumpur, Malaysia \\ Corresponding author email: fahmi982@gmail.com
}

\begin{abstract}
In this work, the optoelectronic parameters of natural dyes extracted from beetroot, red cabbage, walnut leaves, and henna are determined and investigated. These include optical energy gap $\left(E_{g}\right)$, extinction coefficient $(k)$, refractive index $(n)$, dielectric constant $\left(\varepsilon_{r}\right)$ and optical conductivity $\left(\sigma_{r}\right)$. Results showed that cabbage dye exhibited a maximum values of $n=6.08, \varepsilon_{r}=37.18$ and $\sigma_{r}=13.92 \times 10^{-3} \mathrm{~S} / \mathrm{cm}$, while henna dye presented the lowest values of $n=1.19, \varepsilon_{r}=1.5$ and $\sigma_{r}=0.039 \times 10^{-3} \mathrm{~S} / \mathrm{cm}$ compared to the optoelectronic parameters of the other studied samples. The optical absorption in the dyes were found to obey a direct allowed transition between the molecular energy levels. The results presented here can be of a special importance for some potential applications in organic electronics such as organic photovoltaics and sensors.
\end{abstract}

Keywords: optoelectronic parameters, natural dyes, beetroot, cabbage, walnut, henna. 


\section{Introduction}

Nowadays, the use of organic dyes has become the focus of interest in both academia and industry owing to the unique features offered by the dyes including cost-effectiveness, flexibility, electronic tuneability and light weight properties [1-3]. Furthermore, organic dyes show higher absorption coefficient and stronger exciton generation compared to those of the inorganic materials $[4,5]$. Commercial dyes are commonly used in various applications, but they suffer from high cost, supply shortage, heavy metal toxicity and challenging synthesis, which ultimately limit their broad usage compared to the natural dyes. Alternatively, it is possible to overcome these limitations by utilizing natural dyes $[5,6]$.

Dyes are organic compounds which are capable of well absorbing light and retaining their intrinsic color because of the presence of chromophore [7]. Betalains are vacuolar nitrogenous plant pigments having a core structure known as betalamic acid [8]. The best-known source of betalains can be beetroot and cacti [9]. Anthocyanins was also found in a large group of plants which contain red-blue pigments, which is water soluble and $\mathrm{pH}$ sensitive [10]. Red cabbage is one of the main sources of anthocyanins and are chemically glycoside moieties of anthocyanidins derived from the flavylium [11]. In addition to different natural pigments, brown dye and red-orange dye were found in juglone (black Walnuts) and lawsone (henna plant), respectively [12, 13]. A review of literature showed that organic dyes have been successfully utilized in photovoltaic devices [14-20]. Additionally, organic dyes can be of a special interest for the coloration technology and various organic electronics applications. However, before these materials are highly employed for viable applications, a broad investigation on their optoelectronic properties is necessary. Therefore, in the current research work, different natural dyes are extracted through a green method and their optoelectronic parameters are comprehensively investigated. The natural dyes were extracted from 
beetroot, red cabbage, walnut leaf, and henna using specified chemical solvents. This can be a unique detailed study which is performed to determine the most important parameters of the dyes including optical energy gap, refractive index, dielectric constant, and optical conductivity. The results are primarily important when these natural dyes are considered for the application of organic electronics.

\section{Materials and methods}

The Beetroots, Red Cabbages and Henna were purchased in the local markets, while Walnut leaves were collected in a local farm. The raw materials were cut into small pieces and crushed by a grinder followed by drying process to produce very fine powders. To extract the dyes, ethanol, distilled water and a combination of ethanol- $\mathrm{HCl}$ were used. Table 1 shows the designation of the extracted samples and the used solvents for this purpose. In a first approach, $100 \mathrm{ml}$ distilled water was put into a specified beaker then boiled on a hotplate for 2 minutes. Later on, the beakers were transferred, and $50 \mathrm{~g}$ of each sample was inserted into the boiled water, separately. The prepared solutions of sample B1 and C1 were left for 2 hours and then filtered and stored in the refrigerator for 24 hours. In the second approach, $100 \mathrm{ml}$ of ethanol was mixed with $\mathrm{HCl}(0.1)$ with the ratio of 99:1, then $50 \mathrm{~g}$ of samples B2 and C2 was put into the beakers and left for 2 hours. Afterwards, filtration process was performed, and the extracted solutions were stored in the refrigerator for 24 hours. In the third approach, $200 \mathrm{~g}$ of the mashed materials of sample B3 and sample C3 was separately put into a beaker containing distilled water as a solvent, then the beaker was transferred into a hot bath and agitated at $60^{\circ} \mathrm{C}$ for 6 hours. After filtration process, the prepared solutions were put into the oven at $50^{\circ} \mathrm{C}$ until they became a paste. Later, $0.1 \mathrm{~g}$ of the paste was diluted using 10 $\mathrm{ml}$ of distilled water. Finally, a Soxhlet extractor was used for the extraction of dyes with ethanol 
solvent. The same procedure of preparation, filtration and extraction were performed for the other samples.

To extract the dye from walnut leaves, $150 \mathrm{~g}$ of its powder was immersed in $500 \mathrm{ml}$ of ethanol and left for 24 hours in a hot bath at $60^{\circ} \mathrm{C}$. The extracted samples are shown in Figure 1.

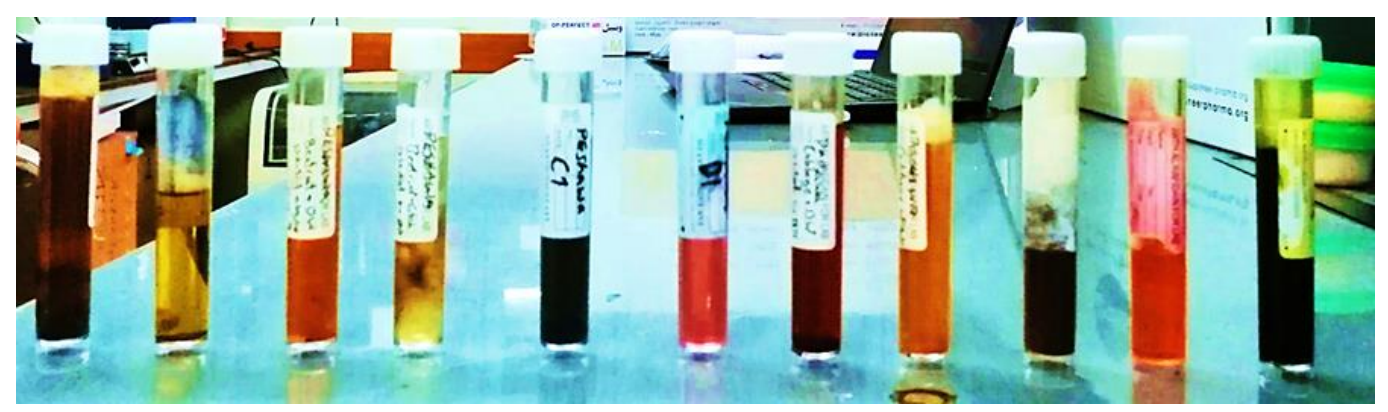

Figure 1: Photo of the extracted dyes.

Table 1: Samples labelling and their extraction description.

\begin{tabular}{|c|c|}
\hline Sample & Description \\
\hline B1 & $50 \mathrm{~g}$ Beetroot $+100 \mathrm{ml}$ Distilled water \\
\hline B2 & 50g Beetroot + $100 \mathrm{ml}$ Ethanol: $\mathrm{HCl}(0.1) \quad(99: 1)$ \\
\hline B3 & $0.1 \mathrm{~g}$ Beetroot paste $+10 \mathrm{ml}$ Distilled water (dilution) \\
\hline B4 & $0.1 \mathrm{~g}$ Beetroot paste $+10 \mathrm{ml}$ Ethanol (diluation) soxhlat method \\
\hline $\mathrm{C} 1$ & $50 \mathrm{~g}$ Cabbage $+100 \mathrm{ml}$ Distilled water \\
\hline $\mathrm{C} 2$ & $50 \mathrm{~g}$ Cabbage $+100 \mathrm{ml}$ Ethanol: $\mathrm{HCl}(0.1)$ \\
\hline $\mathrm{C} 3$ & $0.1 \mathrm{~g}$ Cabbage paste $+10 \mathrm{ml}$ Distilled water (dilution \\
\hline $\mathrm{C} 4$ & $0.1 \mathrm{~g}$ Cabagge paste $+10 \mathrm{ml}$ Ethanol (dilution) soxhlat method \\
\hline E & Sample B1: Sample C1 (1:1) \\
\hline $\mathrm{F}$ & Sample B2: Sample C2 (1:1) \\
\hline $\mathrm{W}$ & $150 \mathrm{~g}$ Walnut $+500 \mathrm{ml}$ Ethanol \\
\hline $\mathrm{H}$ & $100 \mathrm{~g}$ Henna $+200 \mathrm{ml}$ Ethanol \\
\hline
\end{tabular}


Optical absorption studies of the extracted dyes were carried out by using a double beam UV-Vis spectrophotometer (Perkin Elmer, Lambda 25) at room temperature. The extracted samples were inserted into the cuvette for $\mathrm{UV}-\mathrm{V}$ is measurement and their absorbance were recorded in the wavelength range from 200 to $1100 \mathrm{~nm}$. The absorption response of the samples was utilized to determine the optical band gap, optical transition types, optical dispersion parameters, optical dielectric constant, loss factor, and optical conductivity.

\section{Results and discussion}

Figure 2(a-d) shows the absorption coefficient of the dyes in the wavelength range from 200 to $1100 \mathrm{~nm}$ which was calculated from the absorbance data using [21]:

$$
\alpha=\frac{2.303 A}{t}
$$

Where $t$ is the thickness of the cuvette $(\mathrm{q} 1 \mathrm{~cm})$ and $A$ is the absorbance. For the beetroot extract, the absorption spectrum indicated the presence of betalain chromophore [10], as showed multiple peaks of absorption around 475, 525 and $575 \mathrm{~nm}$ due to the presence of mixed pigments. These pigments are yellow betaxanthins (indicaxanthin), red-purple betacyanin (betanin) and betanidin respectively [22]. It was reported that the batlaian dyes can be affected by temperature and $\mathrm{pH}$ [2325]. Figure 2(a) shows the absorption coefficient spectrum of beetroot dye which was extracted by different methods. Results showed that all samples are strongly absorbent in the ultraviolet region, which can be ascribed to the presence of red-violate betalain group [24]. However, different absorption peaks in the visible region have been observed. Sample B2 showed absorption peaks at 486 and $549 \mathrm{~nm}$, which suggested that the extracts contain both betaxanthins and betanidin, and one peak in B3 sample was observed in the visible region at $417 \mathrm{~nm}$, due to the presence of indicaxanthin. However, no peaks were observed in the visible region of samples B1 and B4. 
The absorption spectra of the solutions from red cabbage are shown in Figure 2(b). One can notice that all the samples show a strong absorption in the UV region, which is well matched to the absorption characteristic of anthocyanin dye. This strong absorption in the UV region is due to the conjugated aromatic phenolic double bonds in anthocyanin [26]. Sample C1 and C2 have almost the same absorption peaks around $560 \mathrm{~nm}$, indicating the presence of anthocyanidin [27]. The peaks between $360 \mathrm{~nm}$ to $470 \mathrm{~nm}$ are belong to a non-acylated pigment [28], which are observed in all samples except C2. Figure 2(c) shows the absorption spectrum of a mixed dyes of B1 with $\mathrm{C} 1$ and $\mathrm{B} 2$ with $\mathrm{C} 2$ by 1:1 (V/V) ratio. Interestingly, both samples presented a broad absorption band spanning along the UV and visible regions.

Figure 2-d shows the UV-vis absorption coefficient for both extracted dyes of Walnut leave and Henna. It can be seen that both dyes exhibited similar spectrum due to their comparable chemical structures. The broad absorption in UV region is referred to the presence of intermolecular hydrogen bonds from benzoquinone and electron delocalization of benzoquinone converts a quinone ring. In addition, the UV absorption points the existence of naphthoquinone in the form of hydrogen bonds with carbonyl [29]. However, the absorption in visible region (blue and red light) are corresponding to the mixture of chlorophyll [30], these peaks were also observed for both extracted dyes.
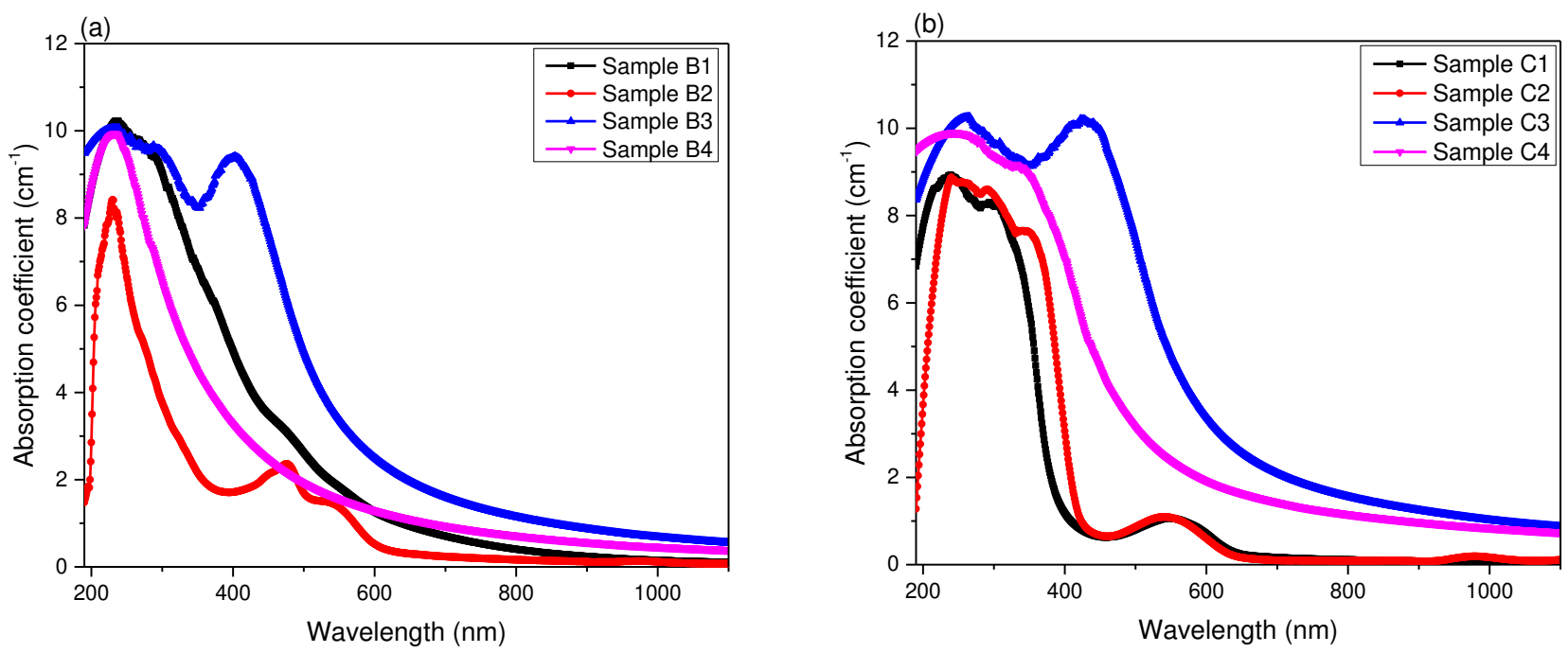

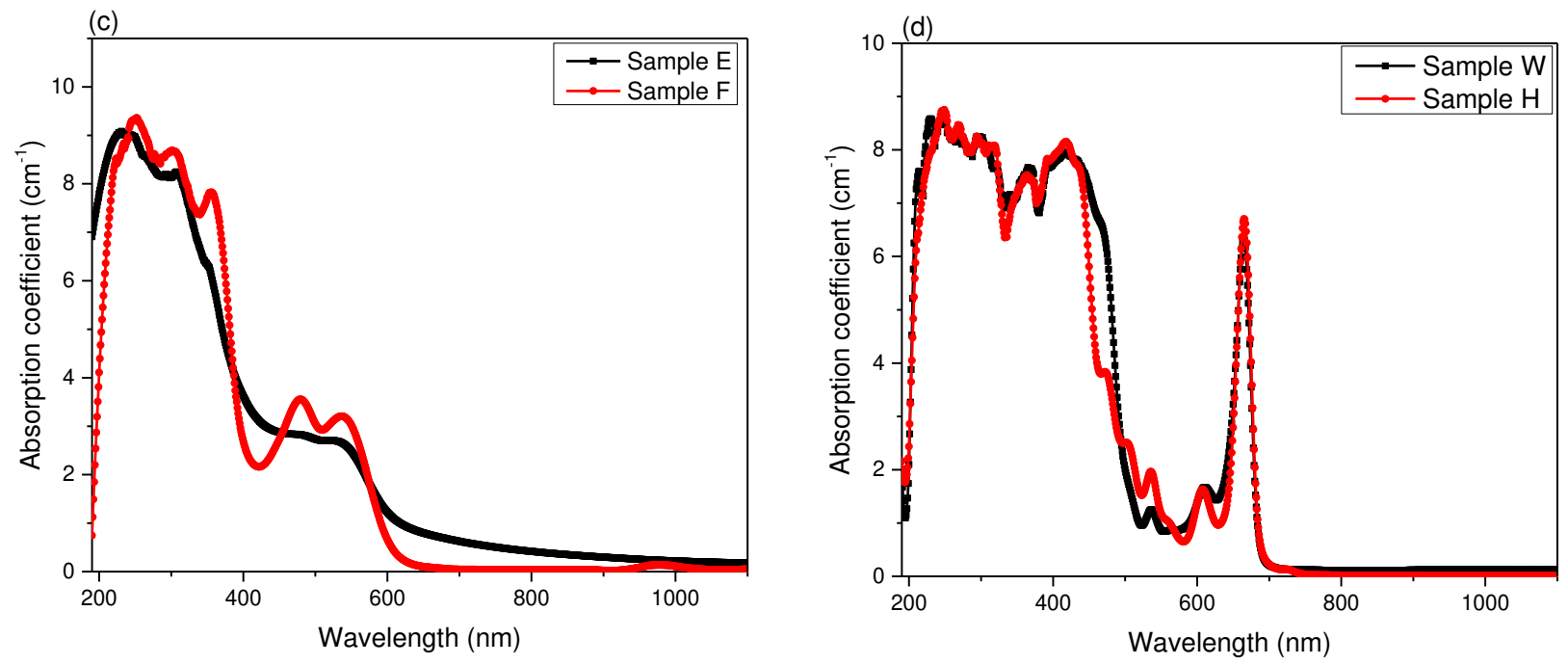

Figure 2: Absorption coefficient spectra of the dyes extracted from (a) Beetroot, (b) Red Cabbage, (c) mixture of beetroot and red cabbage, and (d) Walnuts leaves and Henna.

The measurement of optical energy gap and the nature of electronic transitions in the organic dyes are very crucial for viable optoelectronics applications. The absorption spectra can be used to determine the type of electronic transitions and optical bandgap. To various extends, Tauc's equation was used by the researchers to deduce the optical band gap and the type of electronic transitions governing the absorption process. We have noticed that the use of Tauc's equation to assign the nature of absorption transitions is highly efficient [31, 32] despite its seldom use by the researchers [33-38]. The Tauc's equation can be represented as follows [39]:

$$
\alpha h v=\alpha_{o}\left(h v-E_{g}\right)^{s}
$$

where $\alpha_{o}$ is an energy-independent constant, $E_{g}$ is the energy gap, and the value of $s$ determine the type and nature of transition [40] $s=\frac{1}{2}$ for direct allowed transition,$\frac{3}{2}$ for direct forbidden transitions, respectively, and this exponent value equals to 2 for the indirect allowed transitions 
and 3 is for indirect forbidden transitions, respectively. One can identify the type of transition before estimating the energy gap by taking natural logarithm and derivation of equation 2 to yield:

$$
\frac{d \ln (\alpha h v)}{d(h v)}=\frac{s}{h v-E_{g}} \ldots \ldots \ldots \ldots
$$

Figure 3 (a-d) shows the plot of $\frac{d \ln (\alpha h v)}{d(h v)}$ versus $h v$ for all samples and the approximate value of $h v=E_{g}$ was taken at the peak of the curve. This is because the denominator of the right-hand side of Equation 3 can become minimum where photon energy is approximately equals to the energy gap. Therefore, the estimate value of $E_{g}$ was utilized for plotting $\ln (\alpha h v)$ versus $\ln \left(h v-E_{g}\right)$ and the values of $s$ were determined from the slope of the curves. It was found $\mathrm{s}=\frac{1}{2}$, which indicated the presence of a direct allowed transition between the intermolecular energy bands of the dyes. Afterwards, the precise value of the energy gap was determined by Tauc's equation through plotting $(\alpha h v)^{2}$ as a function of $(h v)$ and taking the extrapolation of linear portion at $(\alpha h v)^{2}=0$. The energy gap location of the samples is depicted in Figure 4 (a-d)and the determined values of $E_{g}$ are shown in Table 2. Results showed that energy gap for each dyes extracted from beetroot and red cabbage are different because they are $\mathrm{pH}$ and temperature sensitive. Noticeably, the dyes extracted from henna and walnut processed a very close value of energy gap due to similarity in their structure.
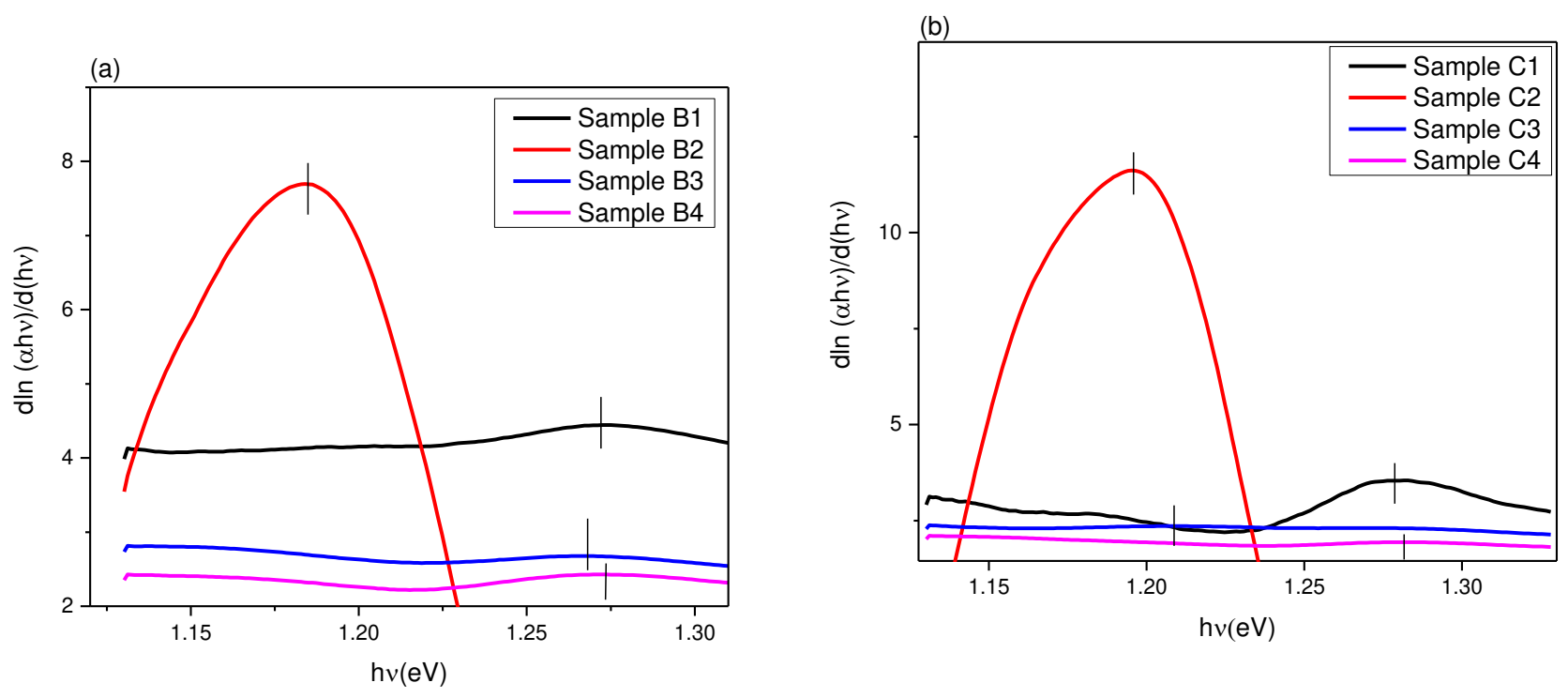

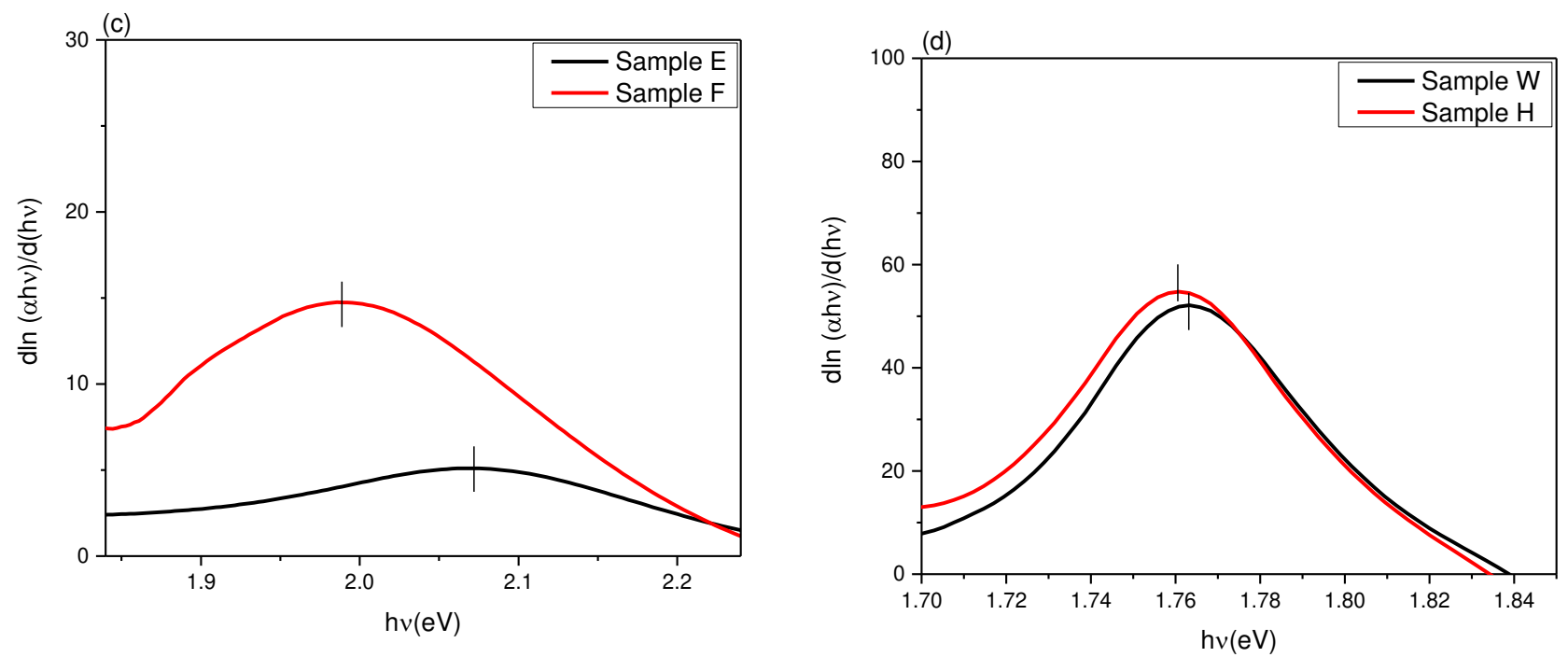

Figure 3: plot of $d \ln (\alpha h v) / d h v$ versus $h v$ for the dyes extracted from (a) Beetroot, (b) Red Cabbage, (c) mixture of beetroot and red cabbage, and (d) Walnuts leaves and Henna
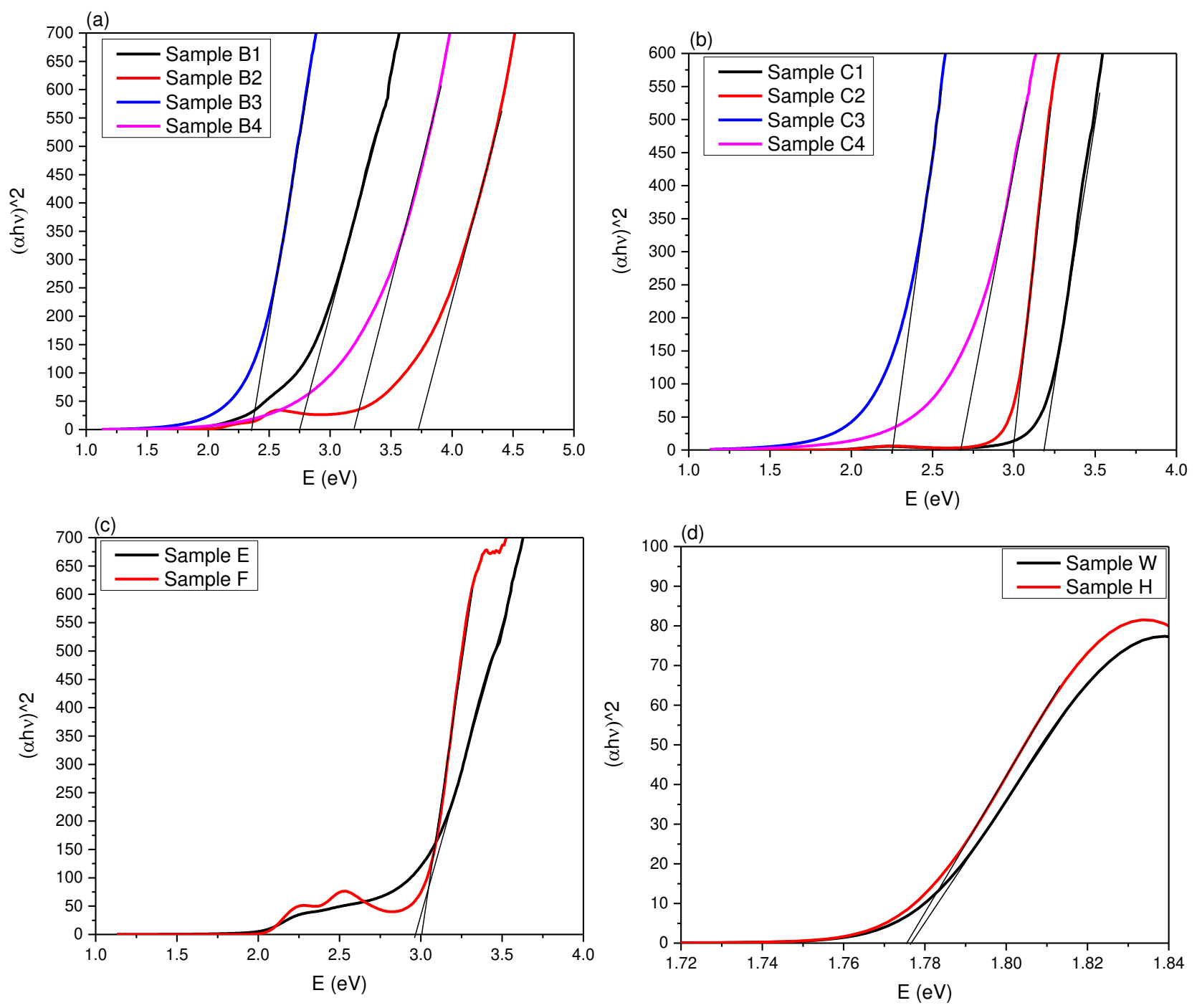
Figure 4: plot of $(\alpha h v)^{2}$ versus $E$ for the dyes extracted from (a) Beetroot, (b) Red Cabbage, (c) mixture of beetroot and red cabbage, and (d) Walnuts leaves and Henna.

Following the absorption coefficient of the samples, refractive index and extinction coefficient are other two optical parameters describe the materials performance. Refractive index tells us how the optical radiation propagates through a medium and it shows that to what extent the speed of light is slowing down inside the medium compared to the speed of light in vacuum. The refractive index is a complex variable, and its imaginary part represents the extinction coefficient, which describes the amount of energy loss in the medium. Both refractive index $(n)$ and extinction coefficient $(\mathrm{k})$ were calculated from the absorbance data using the following equations [21]:

$$
\begin{gathered}
n=\frac{-2(R+1) \pm \sqrt{4 k^{2} R^{2}+16 R-4 k^{2}}}{2(R-1)} \ldots \ldots \ldots \ldots \\
k=\frac{\alpha \lambda}{4 \pi} \ldots \ldots \ldots \ldots \ldots \ldots(5)
\end{gathered}
$$

Where $\mathrm{R}$ is the reflectance and $\alpha$ is the absorption coefficient. They were calculated using the following equations: $\mathrm{R}=1-\mathrm{T}-\mathrm{A}$, where $\mathrm{A}$ is absorbance and $\mathrm{T}$ is transmittance, estimated from $\mathrm{T}=10^{-\mathrm{A}}$ and $\alpha=\frac{2.303 A}{t}$, where $t$ is the thickness of the cuvette. The refractive index of the samples as a function of wavelength in the range of 190 to $1100 \mathrm{~nm}$ is shown in Figure 5 (ad). The variation of refractive index for each sample with wavelength is related to the polarization of the molecules due to the interaction with electromagnetic wave, where the wide dispersion regions describe the polar nature of the samples [33]. At high wavelength, the refractive index reached a plateau region at which the extrapolation of the curve to y-axis gives the static value of 
refractive index, as shown in Table 2. It was observed from Table 2 that the value of $n$ is changed with respect to the method of extraction and the solvent used for extraction. The red cabbage dyes experienced a high value of refractive index (C3), while henna has the lowest value of $n$. Furthermore, Figure 5 (e-h) shows the extinction coefficient $(k)$ as a function of wavelength for all the dye samples. Based on equation 5, it can be seen that the variation in $k$ is almost similar to the corresponding absorption coefficient, as shown in Figure 2 (a-d). The variation of $(k)$ represent the loss of incident electromagnetic wave because of scattering and absorption per unit thickness in the medium[33]. For most of the studies dyes, the maximum values of $(k)$ was observed to be in the UV region.
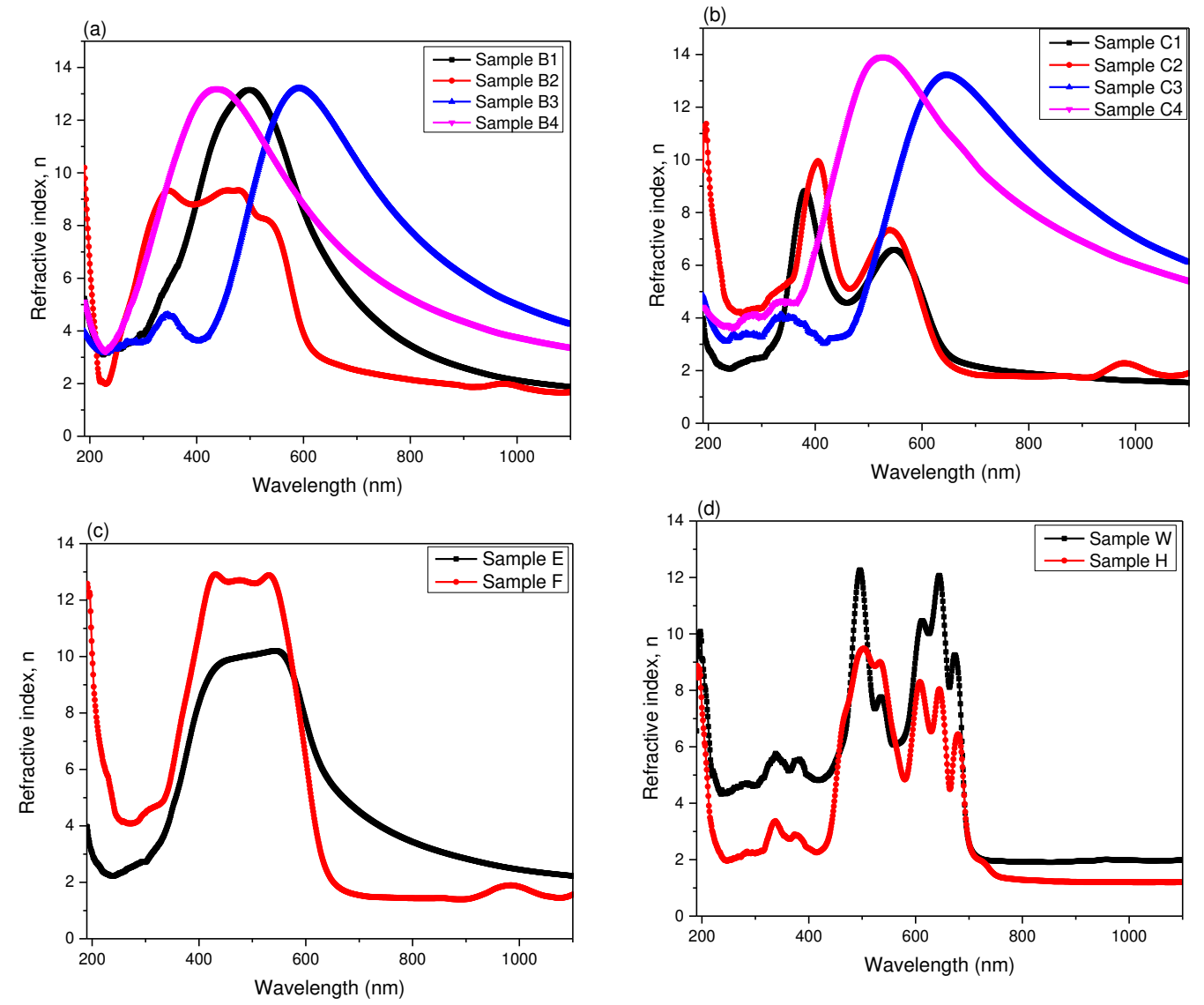

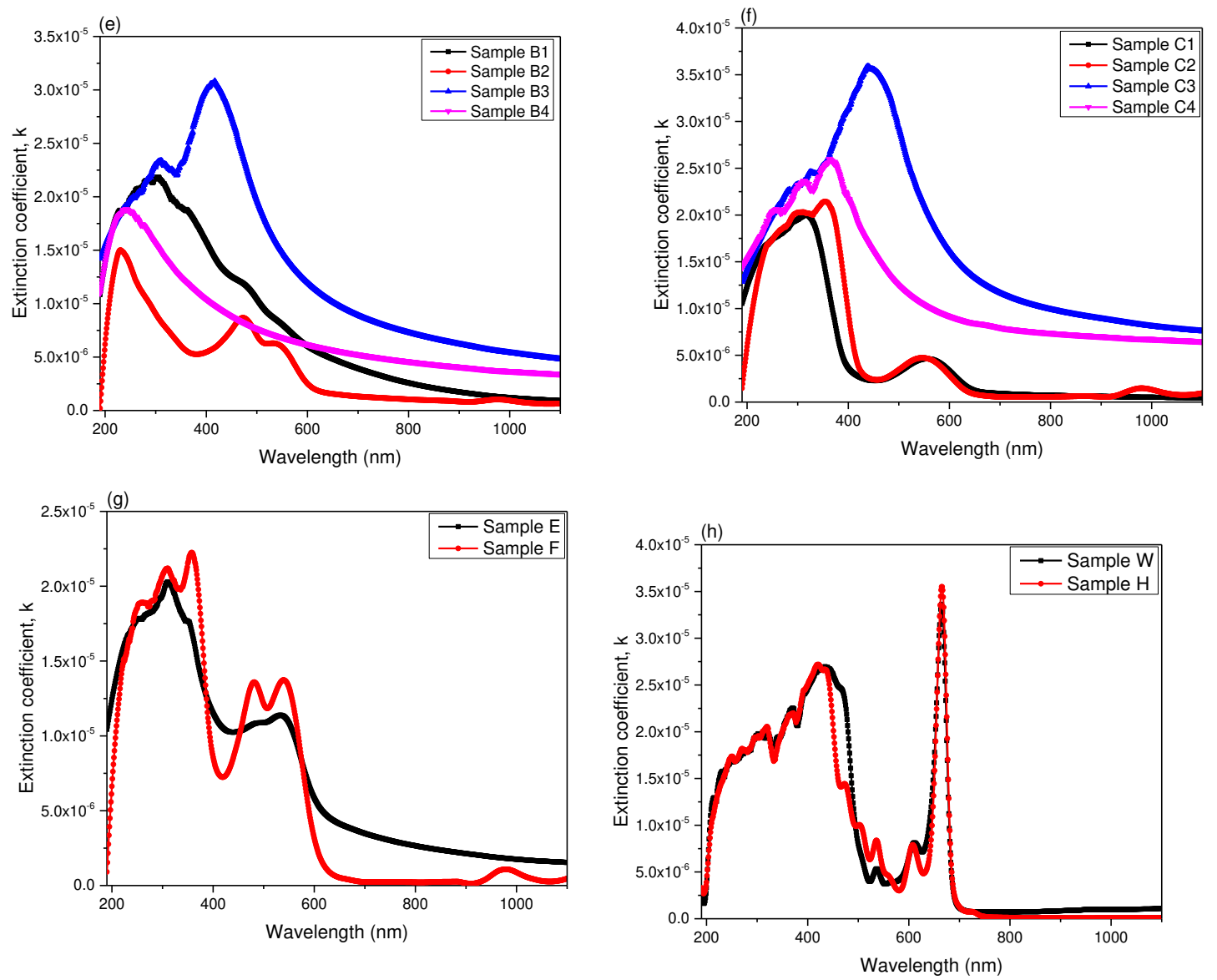

Figure 5: Refractive index and extinction coefficient spectra for the dyes extracted from (a \& e) Beetroot, (b \& f) Red Cabbage, (c \& g) mixture of beetroot and red cabbage, and (d \& h) Walnuts leaves and Henna.

Figure 6 shows the variation of optical dielectric constant over the wavelength of UV to IR region. The response of electrons in the material upon the incident of light is physically represented by optical dielectric constant $(\varepsilon)$, which is a frequency dependent parameter. Since dielectric constant is a complex variable, it has real and imaginary parts and can be mathematically represented by [34]:

$$
\varepsilon=\varepsilon_{1}+i \varepsilon_{2}
$$

Where $\varepsilon_{1}$ represents the real part and $\varepsilon_{2}$ represents the imaginary part of dielectric constant. The real dielectric constant is further represented by equation 7 and it is a measure of to what extent 
the material can be polarized due to the influence of electromagnetic field, while the imaginary part shows the optical loss and is described by equation 8 .

Table 2: The optoelectronics parameters estimated for the extracted dyes.

\begin{tabular}{lllll}
\hline Samples & \multicolumn{1}{c}{$\boldsymbol{n}_{\infty}$} & \multicolumn{1}{c}{$\boldsymbol{\varepsilon}_{\boldsymbol{r}}$} & $\boldsymbol{\sigma}_{\boldsymbol{r}}\left(\frac{S}{\mathrm{~cm}}\right) \times \mathbf{1 0}^{-\mathbf{3}}$ & $\boldsymbol{E}_{\boldsymbol{g}}(\boldsymbol{e V})$ \\
\hline B1 & 1.95 & 3.87 & 0.53 & 2.75 \\
B2 & 1.65 & 2.73 & 0.311 & 3.72 \\
B3 & 4.24 & 18.02 & 6.1 & 2.36 \\
B4 & 3.36 & 11.52 & 3.5 & 3.18 \\
C1 & 1.54 & 2.42 & 0.224 & 3.18 \\
C2 & 1.77 & 3.21 & 0.448 & 2.99 \\
C3 & 6.08 & 37.18 & 13.92 & 2.25 \\
C4 & 5.41 & 29.07 & 10.63 & 2.67 \\
E & 2.25 & 5 & 1.05 & 2.96 \\
F & 1.4 & 2.01 & 0.128 & 3.02 \\
W & 1.9 & 3.65 & 0.57 & 1.776 \\
H & 1.19 & 1.5 & 0.039 & 1.775 \\
\hline
\end{tabular}

The optical loss takes place because of the time lag of $\pi / 2$ between the electric field of the incident photon and the component of electronic or atomic polarization in the optical frequency range [41].

$$
\begin{gathered}
\varepsilon_{1}=n^{2}-k^{2} \ldots \ldots \ldots \ldots \ldots(7) \\
\varepsilon_{2}=2 n k \ldots \ldots \ldots \ldots \ldots(8) \\
\tan \delta=\frac{\varepsilon_{2}}{\varepsilon_{1}} \ldots \ldots \ldots \ldots \ldots(9)
\end{gathered}
$$

Noteworthy, the real part of dielectric constant resembles the refractive index due to small value of $k$. However, the imaginary part is mainly dependent on the absorption coefficient (see Equation 7and 8). The variation in real dielectric constant for all the extracted dyes is shown in Figure 6(ad). It was seen that the peak observed in the UV region is belong to B2 sample, while other samples 
show their characteristic peaks at different wavelengths. It has been reported that the higher value of real dielectric constant indicates the presence of interaction between electron and incident photon in the extracted dyes[35]. It worth to note that the mixed dye of anthocyanin and betalin dye has a broad peak ranging from 400 to $600 \mathrm{~nm}$ (Figure 6-c), whereas walnut leaves and henna has a sharp peak around 500 and $700 \mathrm{~nm}$. The values of real dielectric constants are listed in Table 2 , which were determined from the intersection of extrapolation of the plateau region to y-axis. Dyes extracted from red cabbage, especially C3 presented the maximum value of $\varepsilon_{1}$, while dyes extracted from henna has lowest value. One can notice that the value of $\varepsilon_{1}$ for the extracted dyes with water solvent is greater compared to those extracted with ethanol and $\mathrm{HCl}$ solvent. This might be due to the polar nature of water. In addition to dielectric constant, Figure 6 (e-h) shows the imaginary dielectric constant of all the extracted dyes as a function of wavelength. It can be concluded that the variation of imaginary part of dielectric constant is related to the absorption coefficient of the dyes and their mixtures, as shown in Figure 6-g with a broad peak across UV and visible region. Furthermore, the dissipation factor $(\tan \delta)$ was calculated from equation 8 by dividing the imaginary part to the real part of dielectric function[31]. The spectra of dissipation factors for all samples were presented in Figure 7(a-d), wherein each dye presented different characteristic peaks regardless of whether extracted from beetroot or red cabbage has different peak value and it's depend the type due to the effect of solvents and extraction methods. The anthocyanin dye, which was extracted from red cabbage with distilled water as a solvent (C3), has maximum peak value in the range of 400 to $500 \mathrm{~nm}$ compared to that of betalain dye extracted from beetroot with the same solvent and extraction method. This is ascribed to the rate of absorption of anthocyanin which is greater than that of the betalain dye, whereas their mixture 
showed a broad peak in the UV region. However, walnut leave and henna showed a broad peak expanded to $500 \mathrm{~nm}$ and a sharp peak near $700 \mathrm{~nm}$.
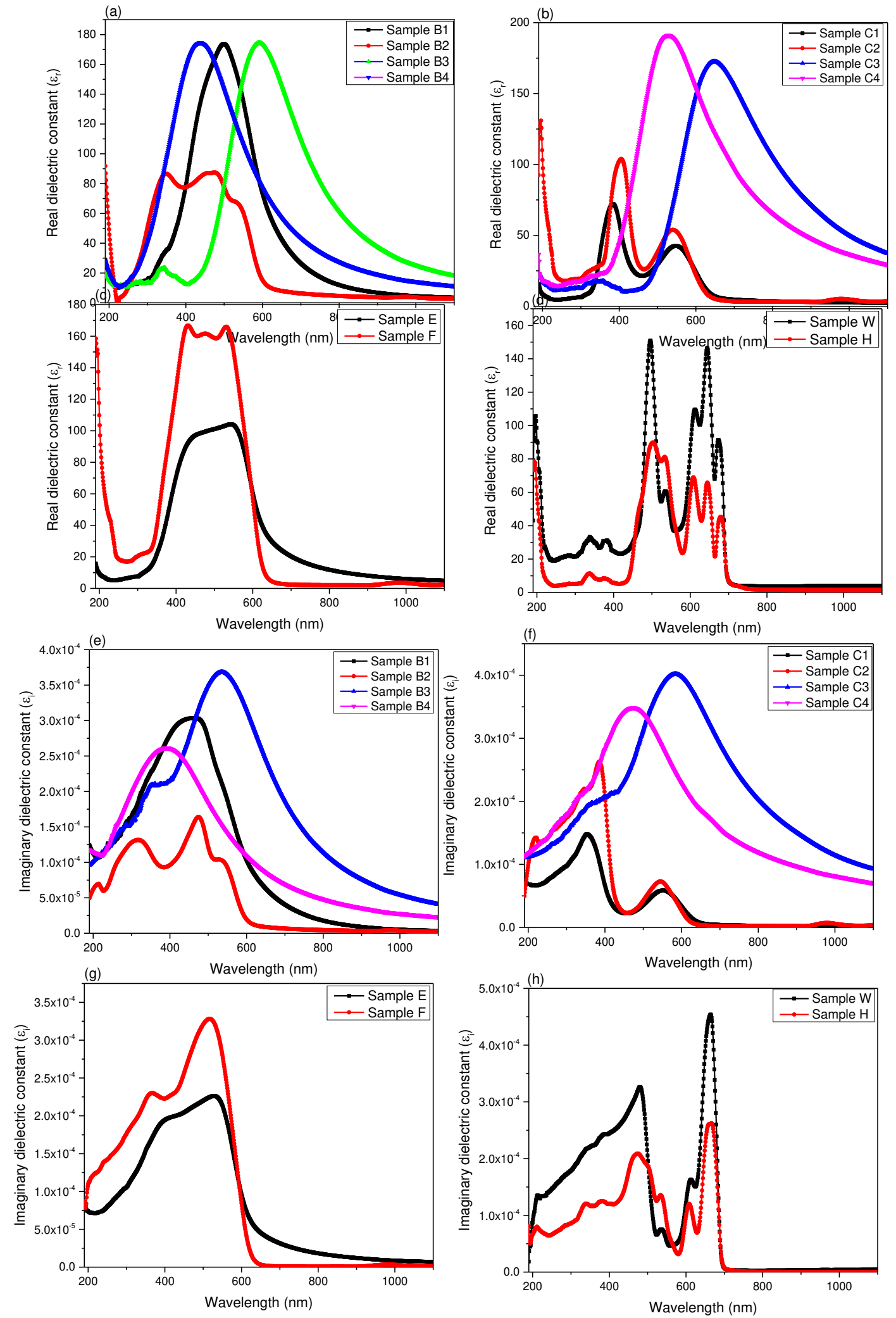
Figure 6: Real and Imaginary optical dielectric constant spectra for all samples of dyes extracted from (a \& e) Beetroot, (b \& f) Red Cabbage, (c \& g) mixture of beetroot and red cabbage, and (d \& h) Walnuts leaves and Henna.
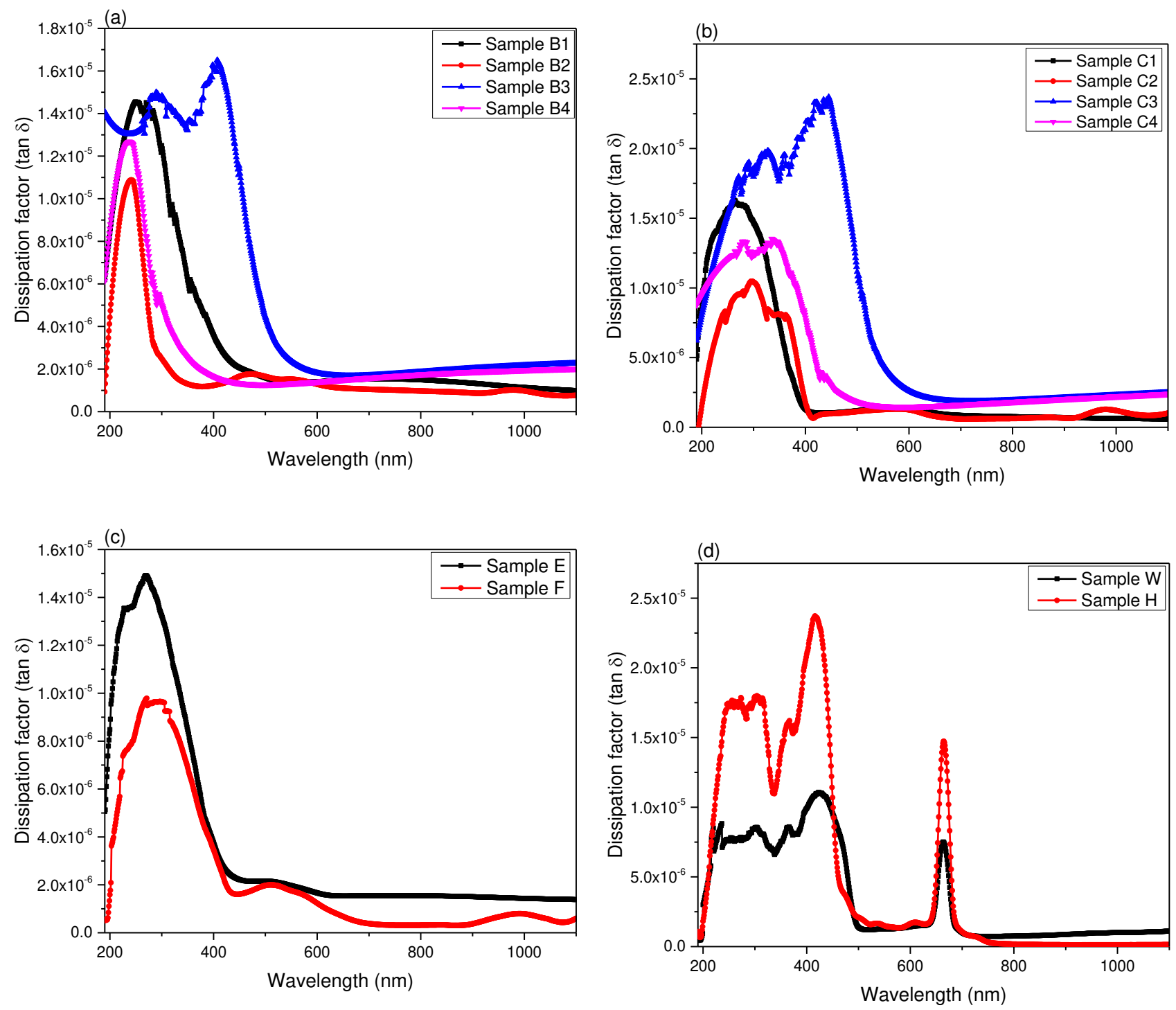

Figure 7: dielectric lost tangent (dissipation factor) spectra for all samples of dyes extracted from (a) Beetroot, (b) Red Cabbage, (c) mixture of beetroot and red cabbage, and (d) Walnuts leaves and Henna. 
To determine the electronic characteristics of a material, optical conductivity is a powerful tool to be used, which describes the response of electron to the absorbed photon. Since the optical conductivity is a complex variable, it is derived from optical dielectric constant[36]:

$$
\begin{aligned}
& \sigma^{*}=\sigma_{r}+i \sigma_{i} \ldots \ldots \ldots \ldots(10) \\
& \sigma_{r}=\omega \varepsilon_{2} \varepsilon_{o} \ldots \ldots \ldots \ldots \ldots(11) \\
& \sigma_{i}=\omega \varepsilon_{1} \varepsilon_{o} \ldots \ldots \ldots \ldots \ldots(12)
\end{aligned}
$$

Where $\sigma_{r}$ and $\sigma_{i}$ are the real and imaginary part of complex optical conductivity respectively, $\omega$ is the angular frequency, and $\varepsilon_{o}$ is the permittivity of free space. One can conclude from equation 10 that the real optical conductivity is related to the imaginary optical dielectric constant and hence depends on the absorption coefficient. Thus, increasing the magnitude of real optical conductivity is directly related to the transport response of electrons which are excited by the absorbed photon energy[42]. In contrast, the imaginary optical conductivity is related to the real optical dielectric constant hence represents the interaction between electromagnetic wave and electrons in the form of polarization. The spectra of real and imaginary optical conductivity as shown in Figure 8 and the value of real optical conductivity are listed in Table 2. It was concluded that anthocyanin dye (C3) has the maximum value, which is $\sigma_{r}=13.92 \times 10^{-3} \mathrm{~S} / \mathrm{cm}$, while the extracted dye from henna has the lowest value of $\sigma_{r}=0.039 \times 10^{-3} \mathrm{~S} / \mathrm{cm}$. In addition, it was seen from the spectra of real conductivity that both betalain and anthocyanin dyes extracted by distilled water as a solvent (B3\&C3) have a broad peak over UV and visible region. The henna and walnut leaves have a broad peak starting from UV region until $500 \mathrm{~nm}$ in visible region and a sharp peak at near IR region. Figure 8 (e-h) shows the imaginary spectra of the optical conductivity and they are similar to real optical dielectric constant spectra. Noticeably, the combination of two dyes together 
has led to a breaded photo-absorption in the UV region, indicating that molecular interactions between their moieties are mostly taken place in the high energy levels.
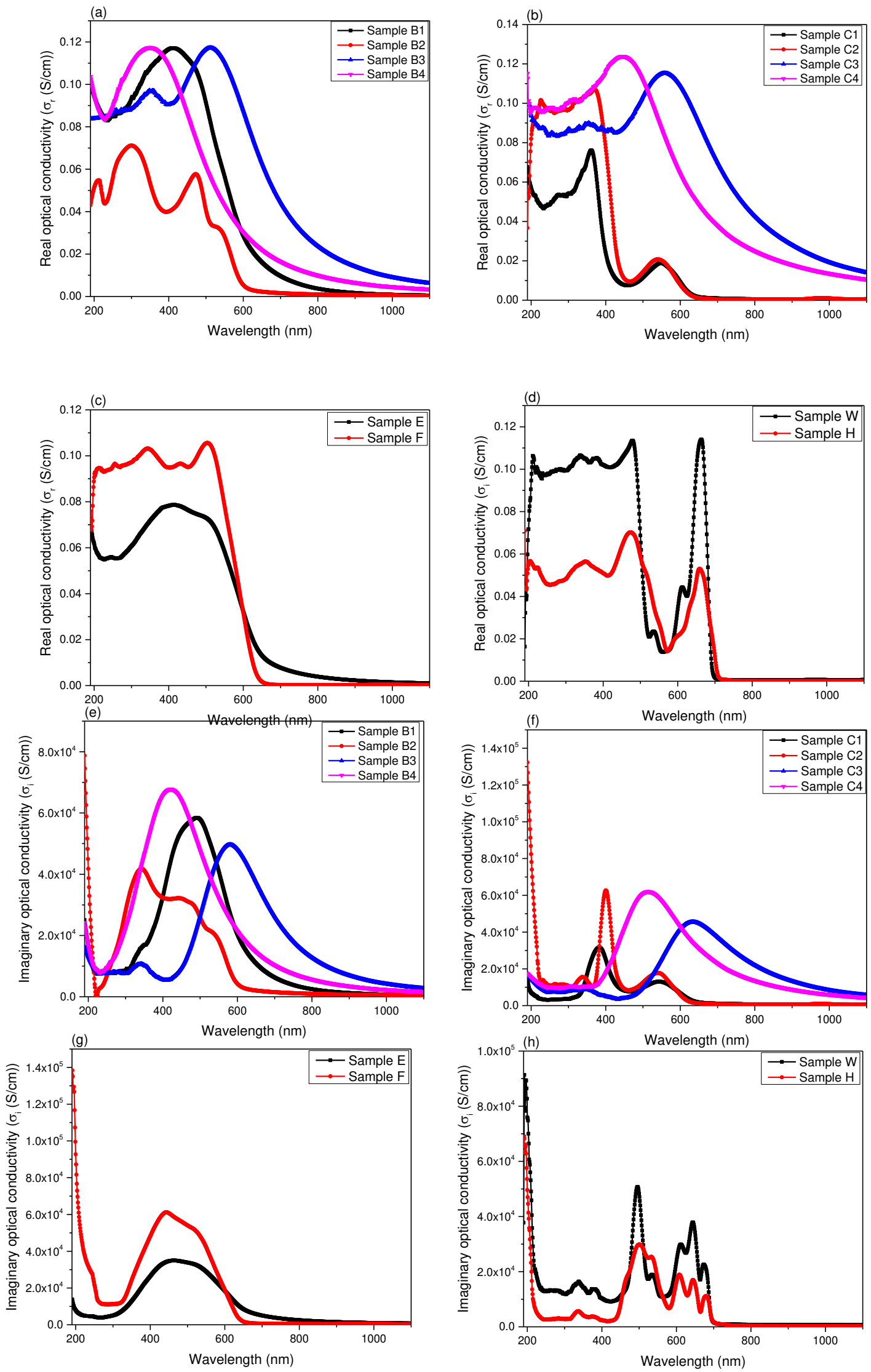
Figure 8: Real and Imaginary optical conductivity spectra for all samples of dyes extracted from (a \& e) Beetroot, (b \& f) Red Cabbage, (c \& g) mixture of beetroot and red cabbage, and (d \& h) Walnuts leaves and Henna.

\section{Conclusions}

Natural dyes were successfully extracted from beetroot, red cabbage, walnut and henna using a facile approach and were broadly characterized. A broad spectrum of optoelectronics parameters of the samples such as absorption coefficient, optical band gap, dispersion parameters, and optical conductivity was investigated. The UV results showed that dyes extracted from beetroot and red cabbage are btalain and anthocyanin derivatives, respectively. However, dyes in henna and walnut have almost similar spectra due to their similar aromatic conjugation double bonds. The cabbage dye showed maximum value of refractive index, real optical dielectric constant, real optical conductivity, while henna showed the minimum value for these parameters. By taking derivatives

of Tauc's equation, the type of electronic transitions was estimated to be direct allowed transition for all the dyes.

\section{Funding statement}

This work did not receive a financial support.

\section{Conflicts of interest}

The authors declare that there is no conflict of interest regarding the publication of this paper.

\section{Author contributions}


Methodology: Peshawa O. Amin; Formal analysis and investigation: Fahmi F. Muhammadsharif, Salah Raza Saeed, and Khaulah Sulaiman; Writing - original draft preparation: Peshawa O. Amin; Writing - review and editing: Fahmi F. Muhammadsharif, Salah Raza Saeed, and Khaulah Sulaiman; Supervision: Fahmi F. Muhammadsharif, Salah Raza Saeed.

\section{Availability of data and material}

The data and material are available within the manuscript.

\section{Compliance with ethical standards}

Not applicable

\section{Consent to participate}

Not applicable

\section{Consent for Publication}

The corresponding author transfers to Springer the non-exclusive publication rights and he warrants that the contribution is original and that he has full power to make this grant. The author signs for and accepts responsibility for releasing this material on behalf of any and all co-authors. This transfer of publication rights covers the non-exclusive right to reproduce and distribute the article, including reprints, translations, photographic reproductions, microform, electronic form (offline, online) or any other reproductions of similar nature. 


\section{Acknowledgement}

The authors gratefully acknowledge the Ministry of Higher Education and Scientific ResearchKurdistan Regional Government-Charmo University_College of Medical and Applied Science

-Department of Applied Physics for the financial support. The authors would like to express their deepest appreciation to University of Sulaimani-College of Science—Department of Physics, and Komar University for Science and Technology for their technical support.

\section{References}

1. Mohammadi, R.H.a.A., Improving optical absorptivity of natural dyes for fabrication of efficient dye-sensitized solar cells. Journal of Theoretical and Applied Physics, 2013. 7: p. 57.

2. Jamali, R.H.a.A., Enhancing the optical absorption of anthocyanins for dye-sensitized solar cells. JOURNAL OF RENEWABLE AND SUSTAINABLE ENERGY, 2015. 7: p. 013120.

3. Trevor M. Grant, T.G., Olivier Dautel, Guillaume Wantzb, and Benoît H. Lessard, Multifunctional ternary additive in bulk heterojunction OPV: increased device performance and stability Journal of Materials Chemistry A, 2013: p. 1-3.

4. Yung-Chung Chen, C.-Y.H., Ryan Yeh-Yung Lin, Kuo-Chuan Ho, and Jiann T. Lin, Materials for the Active Layer of Organic Photovoltaics:Ternary Solar Cell Approach. ChemSusChem 2013. 6: p. 2035.

5. Emre Güzela, B.S.A., Veysel Durmaz, Mert Cesur, Ömer Faruk Tutara, Tuğba Sarı, Mehmet İşleyen, Mehmet Nebioğlu, ilkay Şişman, Photovoltaic performance and photostability of anthocyanins, isoquinoline alkaloids and betalains as natural sensitizers for DSSCs. Solar Energy, 2018. 173: p. 34-41.

6. Bharath K V, A.J., Shoumitra Biswas, T.Ramachandran, K.S.Rajni, Extraction and Absorption Study of Natural Plant Dyes for DSSC. International Journal of ChemTech Research, 2016. 9(01): p. 254258.

7. F. Delgado-Vargas, A.R.J., and O. Paredes-López, Natural Pigments: Carotenoids, Anthocyanins, and Betalains - Characteristics, Biosynthesis, Processing, and Stability. Critical Reviews in Food Science and Nutrition, 2000. 40(3): p. 173-289.

8. Mohammad Imtiyaj Khan, P.G., Plant betalains: Chemistry and biochemistry. Phytochemistry, 2015. 117: p. 267-295.

9. M. Alejandra Guerrero-Rubio, J.E., Francisco García-Carmona, and Fernando Gandía-Herrero, Light Emission in Betalains: From Fluorescent Flowers to Biotechnological Applications. Trends in Plant Science 2019. 11(001).

10. Giuseppe Calogero, J.-H.Y., Alessandro Sinopoli, Gaetano Di Marco, Michael Gra"tzel, Mohammad Khaja Nazeeruddin, Anthocyanins and betalains as light-harvesting pigments for dye-sensitized solar cells. SOLAR ENERGY, 2012. 86: p. 1563-1575.

11. L.C. Kerio, F.N.W., J.K. Wanyoko, , M.K. Rotich, Characterization of anthocyanins in Kenyan teas: Extraction and identification. Food Chemistry, 2012. 131: p. 31-38. 
12. Ali Jahanban-Esfahlan, A.O., Mahnaz Tabibiazar and Ryszard Amarowicz, A Comprehensive Review on the Chemical Constituents and Functional Uses of Walnut (Juglans spp.) Husk International Journal of Molecular Science 2019. 20: p. 3920.

13. Henari, F.Z., Optical Nonlinear Properties and Optical Switching of Henna (Lawson) Films International Journal of Thin Films Science and Technology, 2012. 1(2): p. 55-60.

14. Paudel, P.N., Pandey, A, Shrestha, R.R., Neupane, A.,Lamichhane, P.,Adhikari, R.,4Gyawali, R. and Kafle, B.P., Optical properties of natural dyes: prospect of application in dye sensitized solar cells (DSSCs) and organic light emitting diodes (OLEDs). Food Research 2018. 2(5): p. 429-436.

15. Dhafina WA, D.M., Salleh H, The sensitization effect of anthocyanin and chlorophyll dyes on optical and photovoltaic properties of zinc oxide based dye-sensitized solar cells. Optik, 2019.

16. Adedokun O, S.Y., Awodugba AO, Solvent Dependent Natural Dye Extraction and its Sensitization Effect for Dye Sensitized Solar Cells. Optik, 2018. 6: p. 64.

17. Giuseppe Calogero, G.D.M., Stefano Caramori, Silvia Cazzanti, Roberto Argazzi and Carlo Alberto Bignozzi, Natural dye senstizers for photoelectrochemical cells. Energy \& Environmental Science, 2009. 2: p. 1162-1172.

18. Satoshi Honda, T.N., Hideo Ohkita, Hiroaki Benten, and Shinzaburo Ito, Improvement of the LightHarvesting Efficiency in Polymer/Fullerene Bulk Heterojunction Solar Cells by Interfacial Dye Modification. APPLIED MATERIALS \& INTERFACES, 2009. 1(4): p. 804-810.

19. Satoshi Honda, H.O., Hiroaki Benten and Shinzaburo Ito, Multi-colored dye sensitization of polymer/fullerene bulk heterojunction solar cells. Chem. Commun., 2010. 46: p. 6596-6598.

20. Shuhua Zhang, M.N.S., Feng Liu, Zhongqiang Zhang, Qin Hu, Thomas P. Russell, Minmin Shi, Chang$\mathrm{Zhi} \mathrm{Li}$, and Hongzheng Chen, Efficient and 1,8-diiodooctane-free ternary organic solar cells fabricated via nanoscale morphology tuning using small-molecule dye additive Nano Research, 2017.

21. Fahmi Fariq Muhammad, S.B.A., Sarkawt A. Hussein, Effect of the dopant salt on the optical parameters of PVA:NaNO3 solid polymer electrolyte. J Mater Sci: Mater Electron, 2015. 26: p. 521529.

22. Mfoniso I. Udonkang, I.J.I., Affiong N. Ukorebi, Faith Effiong, Uwem Akpan, Iya E. Bassey, Spectrophotometry, Physiochemical Properties, and Histological Staining Potential of Aqueous and Ethanol Extracts of Beetroot. Biomedicine Hub 2018. 3: p. 492828.

23. D. Sengupta, B.M., K. Mukherjee, Visible light absorption and photo-sensitizing properties of spinach leaves and beetroot extracted natural dyes. Spectrochimica Acta Part A: Molecular and Biomolecular Spectroscopy, 2015. 3: p. 120.

24. Kasim Uthman Isah, U.A., Adamu Idris, Mohammed Isah Kimpa, Uno Essang Uno, Muhammed Muhammed Ndamitso, Noble Alu, Betalain pigments as natural photosensitizers for dyesensitized solar cells: the effect of dye $\mathrm{pH}$ on the photoelectric parameters. Mater Renew Sustain Energy 2014. 3: p. 39.

25. A. DUMBRAVĂ, I.E., C. I. OPREA, A. GEORGESCU, M. A. GîRJU, TOWARD A MORE EFFICIENT UTILISATION OF BETALAINS AS PIGMENTS FOR DYE-SENSITIZED SOLAR CELLS. Digest Journal of Nanomaterials and Biostructures 2012. 7(1): p. 339-351.

26. Stewart, G.S.W.a.G.R., Do anthocyanins play a role in UV protection of the red juvenile leaves of Syzygium? Journal of Experimental Botany, 1998. 49(325): p. 1447-1450.

27. Zularif Zolkepli, A.L., Piyasiri Ekanayake, and Kushan Tennakoon, Efficiency Enhancement of Cocktail Dye of Ixora coccinea and Tradescantia spathacea in DSSC. Journal of Biophysics, 2015.

28. Neda Ahmadiani, R.J.R., Thomas M. Collins, M. Monica Giusti, Molar absorptivity (e) and spectral characteristics of cyanidin-based anthocyanins from red cabbage. Food Chemistry, 2016. 197: p. 900-906. 
29. L Qadariyah, N.A., A Q Syafa'atullah, D S Bhuana, M Mahfud, The Extraction of Natural Dyes from Henna Leaves (Lawsonia Inermis L.) by Ultrasound-assisted Method. IOP Conf. Series: Materials Science and Engineering, 2019. 543.

30. Saif Saadaoui, M.A.B.Y., Moufida Ben Karoui, Rached Gharbi, Emanuele Smecca, Vincenzina Strano, Salvo Mirabella, Alessandra Albert, and Rosaria A. Puglisi, Performance of natural-dyesensitized solar cells by ZnO nanorod and nanowall enhanced photoelectrodes. Beilstein Journal of Nanotechnology, 2017. 8: p. 287-295.

31. Fahmi Fariq Muhammad, K.S., Utilizing a simple and reliable method to investigate the optical functions of small molecular organic films - Alq3 and Gaq3 as examples. Measurement, 2011. 44: p. 1468-1474.

32. FAHRETTIN YAKUPHANOGLU, H.E., Refractive index dispersion and analysis of the optical constatnts of an ionomer thin film Otica applicata 2005(4).

33. Shujahadeen B. Aziz, O.G.A., Ahang M. Hussein, Rebar T. Abdulwahid, Mariwan A. Rasheed, Hameed M. Ahmed, Sarbast W. Abdalqadir, Awara R. Mohammed, Optical properties of pure and doped PVA:PEO based solid polymer blend electrolytes: two methods for band gap study. J Mater Sci: Mater Electron 2017. 28: p. 7473-7479.

34. Peshawa O. Amin, A.J.K., Majida A. Ameen, Rebar T. Abdulwahid, Structural and optical properties of thermally annealed TiO2-SiO2 binary thin films synthesized by sol-gel method. Journal of Materials Science: Materials in Electronics 2018. 29: p. 16010-16020.

35. T.S. Soliman, S.A.V., Effect of Fe nanoparticles on the structure and optical properties of polyvinyl alcohol nanocomposite films. Journal of Non-Crystalline Solids, 2019. 519: p. 119452.

36. G.B. Sakr, I.S.Y., M. Fadel, S.S. Fouad, N. Romcevic, Optical spectroscopy, optical conductivity, dielectric properties and new methods for determining the gap states of CuSe thin films. Journal of Alloys and Compounds, 2010. 507: p. 557-562.

37. S. Sudhahar, M.K.K., A. Silambarasan, R. Muralidharan and R. Mohan Kumar, Studies on Structural, Spectral, and Optical Properties of Organic Nonlinear Optical Single Crystal: 2-Amino-4,6dimethylpyrimidinium p-Hydroxybenzoate. Journal of Materials, 2013.

38. Alwan, T.J., Refractive Index Dispersion and Optical Properties of Dye Doped Polystyrene Films. Malaysian Polymer Journal, 2010. 5(2): p. 204-213.

39. Tauc, J., Optical properties and electronic structure of amorphouse Ge and Si Materials Research Bulletin, 1968. 3: p. 37-46.

40. Davis, E.A. and N.F. Mott, Conduction in non-crystalline systems V. Conductivity, optical absorption and photoconductivity in amorphous semiconductors. Philosophical Magazine A, 1970. 22(179): p. 903-922.

41. Sonal, A.S., Sanjeev Aggarwal, Optical investigation of soda lime glass with buried silver nanoparticles synthesised by ion implantation. Journal of Non-Crystalline Solids, 2018. 1: p. 38.

42. Abdullah, A.Q., Surface and Volume Energy Loss, Optical Conductivity of Rhodamine 6G dye (R6G) Chemistry and Materials Research 2013. 3(10). 